\title{
EFFECTS OF SURFACE CHARGE ON THE CONDUCTANCE OF THE GRAMICIDIN CHANNEL
}

\author{
H.-J. APELL, E. BAMBERG and P. LÄUGER \\ Department of Biology, University of Konstanz, D-7750 Konstanz (F.R.G.) \\ (Received August 31st, 1978) \\ (Revised manuscript received December 5 th, 1978)
}

Key words: Surface charge; Conductance; Gramicidin channel

\section{Summary}

The electric conductance of the cation-permeable gramicidin channel in negatively charged phosphatidylserine membranes has been studied. At low electrolyte concentrations the single-channel conductance is much larger in the negatively charged membrane than in a neutral membrane. This enhancement of conductance is in agreement with theoretical expectations, although a complete description of the salt concentration dependence of conductance was not possible. The results of these experiments may be compared with previous studies of a negatively charged gramicidin analog ( $O$-pyromellityl gramicidin). It is found that the electrostatic effect on the conductance is much larger for a neutral channel embedded in a negatively charged lipid than for the negatively charged $O$-pyromellityl analog (with three charges at the channel mouth) embedded in a neutral lipid.

\section{Introduction}

Ion transport across biological membranes may be influenced by an electrostatic potential difference between membrane surface and aqueous solution [1], such surface potentials may result from net charges on bound proteins or from the presence of lipids with cationic or anionic head-groups. The fixed charge tends to accumulate oppositely charged ions (counterions) and to deplete ions of the same sign (co-ions) in the aqueous solution near the surface. In this way the transport rate of counterions is enhanced and the transport rate of co-ions is diminished. These effects of surface charges have been demonstrated with lipopholic ions [2-6] and with macrocyclic ion carriers [7,8] using planar lipid bilayer membranes. For instance it has been found that the conductance of a phosphatidylethanolamine membrane in the presence of the positively charged 
nonactin- $\mathrm{K}^{+}$complex is high at $\mathrm{pH} 10.9$ where the membrane has a negative surface charge and low at $\mathrm{pH} 2.4$ where the membrane is positively charged [7]. The magnitude of the conductance change depends on the ionic strength of the solution in a manner predicted by the Gouy-Chapman theory of the diffuse double-layer [1]. The effects of surface charge have also been discussed for transport systems such as monazomycin [9] or amphotericin B [10] which presumably form pore-like channels in the lipid bilayer [11,12]. Evidence for the influence of surface charges on the conductance of ion channels in nerve has been obtained from studies of $\mathrm{pH}$ effects on the axon [14-16].

In the following we describe experiments in which electrostatic effects of surface charges on a well-characterized ion channel, the gramicidin A channel, have been studied. Gramicidin A is a hydrophobic pentadecapeptide which forms dimeric channels permeable to small monovalent cations (for a recent review, see Ref. 13). In this case the conductance of the single channel can be directly obtained from records of current fluctuations in the presence of minute amounts of the channel-forming peptide. The results of these studies show that the single-channel conductance is strongly affected by the presence of fixed charges on the lipid surrounding the channel.

\section{Materials and Methods}

Gramicidin A (consisting of a mixture of the valine and isoleucine analogues) was kindly provided by Dr. E. Gross (Bethesda). Diphytanoyl phosphatidylserine and diphytanoyl phosphatidylcholine (L-1,2-diphytanoyl-3-phosphatidylcholine) were synthesized by K. Janko [17]; the purity of these lipids was checked by thin-layer chromatography. All other reagents were analytical grade. Optically black lipid membranes were formed in the usual way [18] on a hole in the septum of a thermostated teflon cell. The membrane area was about $5 \cdot 10^{-4} \mathrm{~cm}^{2}$ and the temperature $25^{\circ} \mathrm{C}$. The membrane-forming solution contained $1-2.5 \%(\mathrm{w} / \mathrm{v})$ of the lipid in $n$-decane. The single-channel conductance of gramicidin was measured as described previously $[19,20]$. The records of conductance fluctuations were analyzed either by counting the number of steps within a given conductance interval or by digitizing and analyzing data, using a PDP 11/40 computer [21].

\section{Results}

The conductance $\Lambda$ of the gramicidin channel was determined from the size of the discrete conductance fluctuations observed in the presence of very small amounts of gramicidin. In Fig. 1 the channel conductance is plotted as a function of the concentration of the permeable ion $\left(\mathrm{Cs}^{+}\right)$for neutral diphytanoyl phosphatidylcholine membranes and for negatively charged phosphatidylserine membranes. Fig. 1 shows that for neutral membranes the channel conductance increases almost linearly with increasing $\mathrm{Cs}^{+}$concentration and reaches a maximum in the vicinity of $\left[\mathrm{Cs}^{+}\right] \approx 2 \mathrm{M}$. With negatively charged membranes the conductance shows only a slight concentration dependence for ion concentrations lower than $0.05 \mathrm{M}$ and becomes virtually concentration independent above. In order to exclude effects of multivalent cations, $10^{-4}-5 \cdot 10^{-4} \mathrm{M}$ 


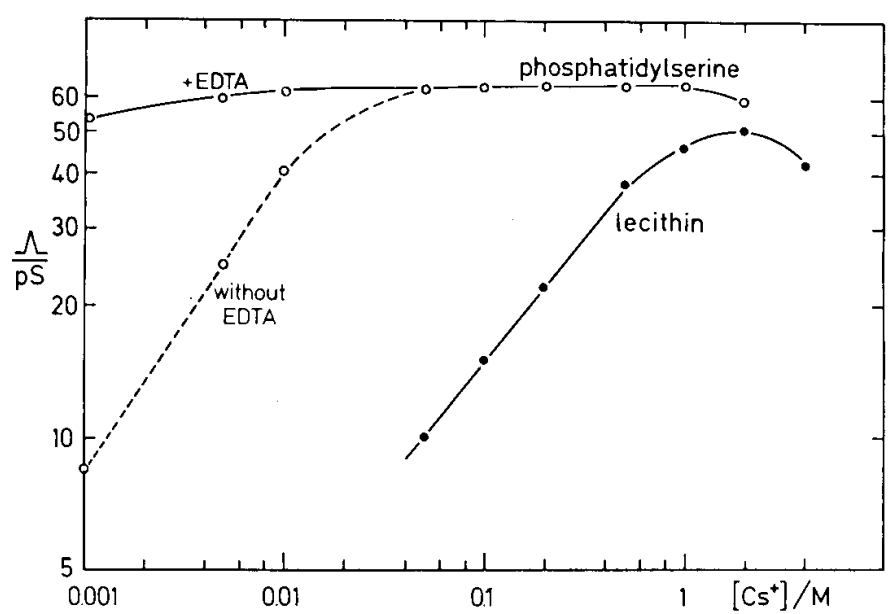

Fig. 1. Conductance $\Lambda$ of the gramicidin channel in membranes made from diphytanoyl phosphatidylserine or diphytanoyl phosphatidylcholine as a function of concentration of the permeable ion $\left(\mathrm{Cs}^{+}\right)$in water $\left(1 \mathrm{pS}=10^{-12} \mathrm{~S}=10^{-12} \Omega^{-1}\right.$ ). The aqueous solutions were buffered with $10^{-4} \mathrm{M}$ Tris to a $\mathrm{pH}$ of 9.1 in the case of phospjatidylserine membranes. The experiments with phosphatidylcholine membranes were done with unbuffered solutions, $\mathrm{pH} \approx 6-8$. If added to the aqueous solution the final concentration of EDTA was $10^{-4} \mathrm{M}$ at $\left[\mathrm{Cs}^{+}\right]<50 \mathrm{mM}$, otherwise $5 \cdot 10^{-4} \mathrm{M} \Lambda$ was determined from the peak in the amplitude distribution of current fluctuations by dividing through the applied voltage $(50-100 \mathrm{mV})$.

ethylenediaminetetraacetic acid (EDTA) was added to the electrolyte. Without EDTA the single channel conductance considerably decreased at low $\mathrm{CsCl}$ concentrations (Fig. 1). This effect presumably results from binding of multivalent cations which may be present as trace impurities in the aqueous solutions. In diphytanoyl phosphatidylcholine and in diphytanoyl phosphatidylserine membranes the mean lifetime of the channel was nearly the same, about $1-2 \mathrm{~s}$ at $\left[\mathrm{Cs}^{+}\right]=1 \mathrm{M}$.

Values of the single-channel conductance in membranes made from mixtures of phosphatidylserine and phosphatidylcholine are given in Table I. Table I

\section{TABLE 1}

Single-channel conductance $\Lambda$ (in pS) in membranes made from mixtures of diphytanoyl phosphatidylserin (PS) and diphytanoyl phosphatidylcholine (PC). PS/PC is the molar ratio of the lipids in the membrane-forming solution. The solutions were unbuffered $(\mathrm{pH} \approx 6)$ except for the experiments with pure membranes $(0.1 \mathrm{mM}$ Tris, $\mathrm{pH} 9.1)$. The effect of $\mathrm{pH}$ on $\Lambda$ was small at salt concentrations above $5 \mathrm{mM}$ in the range between $\mathrm{pH} 6$ and $9 . \Lambda$ was determined from the peak in the amplitude distribution of current fluctuations by dividing through the applied voltage $(50-100 \mathrm{mV})$

\begin{tabular}{|c|c|c|c|c|c|}
\hline Electroly te & PS * & PS & $\begin{array}{l}\mathrm{PS} / \mathrm{PC} \\
(1: 1)\end{array}$ & $\begin{array}{l}\mathrm{PS} / \mathrm{PC} \\
(1: 3)\end{array}$ & $\mathrm{PC}$ \\
\hline $5 \mathrm{mM} \mathrm{CsCl}$ & 28 & 58 & 35 & 32 & - \\
\hline $10 \mathrm{mM} \mathrm{CsCl}$ & 42 & 60 & 40 & 37 & $\approx 2$ \\
\hline $50 \mathrm{mM} \mathrm{CsCl}$ & 64 & 64 & 49 & 47 & 10 \\
\hline $100 \mathrm{mM} \mathrm{CsCl}$ & 65 & 65 & 52 & 49 & 15 \\
\hline $100 \mathrm{mM} \mathrm{CsCl}+1 \mathrm{mM} \mathrm{CaCl}_{2}$ & 19 & - & 19 & 20 & 15 \\
\hline $1 \mathrm{M} \mathrm{CsCl}$ & 66 & 66 & 54 & 50 & 46 \\
\hline
\end{tabular}

* Without EDTA. 
also contains the conductance values observed in the presence of $1 \mathrm{mM} \mathrm{Ca}^{2+}$. Interestingly it is found that in $\left(1 \mathrm{mM} \mathrm{Ca}^{2+}+100 \mathrm{mM} \mathrm{Cs}^{+}\right)$the conductance is virtually the same, irrespective of the phosphatidylserine/phosphatidylcholine ratio. As a divalent cation, $\mathrm{Ca}^{2+}$ is strongly accumulated in the diffuse doublelayer and is more effective in reducing the negative surface potential than univalent cations [7,8]. Furthermore, $\mathrm{Ca}^{2+}$ exerts a direct blocking effect on the gramicidin channel [34]. Under the conditions of these experiments, presumably both the reduction of surface potential as well as the direct blocking effect had an influence on the channel conductance.

\section{Discussion}

The experimental results presented above demonstrate that the conductance of the gramicidin channel is strongly influenced by the presence of fixed charges on the lipid. At low ionic strength the cation-permeable channel has a much higher conductance in the negatively charged membrane than in the neutral membrane. At higher ion concentrations the channel conductance approaches similar saturation values in both cases. This saturation effect probably results from the fact that the channel can accomodate only a limited number of ions [19,22-25]. The behaviour of a neutral channel embedded in a negatively charged membrane, which is described here, may be compared with previous studies on a charged gramicidin analog (O-pyromellityl gramicidin) [26]. In the previous case, three negative charges were fixed at the channel mouth whereas the surrounding lipid was neutral. The results of both studies are compared in Fig. 2. It is seen that the enhancement of conductance is much larger when a neutral channel is surrounded by negatively charged lipid than in the case where a pyromellityl residue with three negative charges is attached to the channel mouth.

The experimental findings with negatively charged membranes may be compared with the Gouy-Chapman theory of the diffuse double-layer [27]. Under conditions where $\Lambda$ is proportional to the cation concentration at the surface of the membrane, the ratio $\Lambda_{\mathrm{G}}$ (phosphatidylserine) $/ \Lambda_{\mathrm{G}}$ (phosphatidylcholine) of Fig. 2 is predicted by the Gouy-Chapman theory to be

$\frac{\Lambda_{\mathrm{G}}(\text { phosphatidylserine })}{\Lambda_{\mathrm{G}}(\text { phosphatidylcholine })}=\exp \left(-\psi_{\mathrm{s}} F / R T\right)$

where $\psi_{\mathrm{s}}$ is the electrical potential difference between the membrane surface and the solution far from the surface, $F$ the Faraday constant, $R$ the gas constant, and $T$ the absolute temperature. Implicit in Eqn. 1 is the assumption that the only effect of the negatively charged lipid on $\Lambda$ consists in an increase of surface concentration of permeable cations; thus, any difference in the dipolar potential [28] of lecithin and phosphatidylserine membranes is neglected. $\psi_{\mathrm{s}}$ is related to the surface charge density $\sigma$ by the relation

$\psi_{\mathrm{s}}=2 \frac{R T}{F} \ln \left[\frac{\sigma}{\sigma_{0}}+\sqrt{\left(\frac{\sigma}{\sigma_{0}}\right)^{2}}+1\right]$

$\sigma_{0} \equiv \sqrt{8 \epsilon_{0} \epsilon R T c}$ 


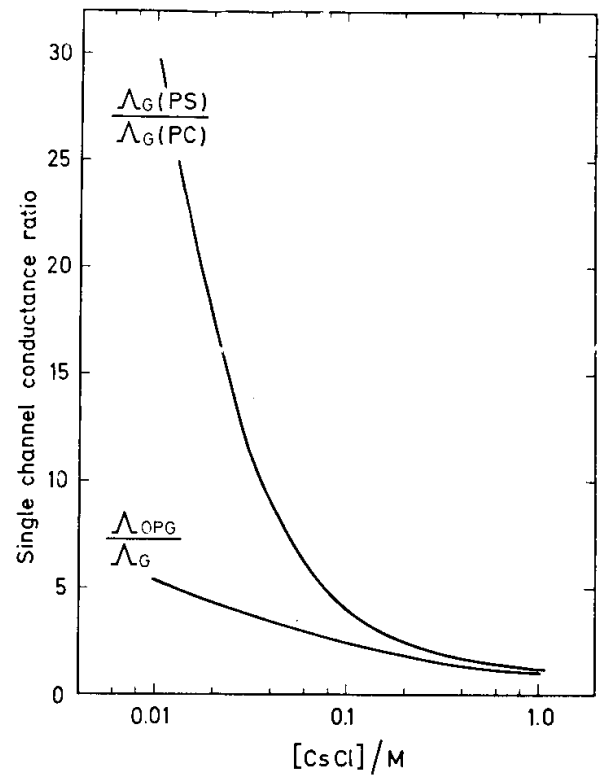

Fig. 2. Conductance ratio as a function of ion concentration. Lower curve ratio of single-channel conductances of negatively charged $O$-pyromellity gramicidin ( $O-P G$ ) and of neutral gramicidin (G) in a membrane made from a neutral lipid (monoolein). Data taken from Ref. 25. Upper curve, ratio of singlechannel conductances of neutral gramicidin in a negatively charged phosphatidylserine (PS) and a neutral phosphatidylcholine (PC) membrane.

$\epsilon_{0}=8.85 \cdot 10^{-12} \mathrm{C} \cdot \mathrm{V}^{-1} \cdot \mathrm{m}^{-1}$ is the permittivity of free space, $\epsilon$ the dielectric constant of water and $c$ the concentration of the $1: 1$ electrolyte. The surface concentration $c_{\mathrm{s}}$ of the univalent cation is given by the Boltzmann relation

$c_{\mathrm{s}}=c \cdot \exp \left(-\psi_{\mathrm{s}} F / R T\right)$

Eqns. 2-4 together have the interesting property that the surface concentration approaches a finite value

$c_{\mathrm{s}}^{0}=\frac{\sigma^{2}}{2 \epsilon_{0} \epsilon R T}$

in the limit of $c \rightarrow 0$ (for $c>0, c_{\mathrm{s}}$ is always larger than $c_{\mathrm{s}}^{0}$ ). The charge density $\sigma$ may be estimated from the area/molecule in a densely packed phospholipid monoplayer (approx. $0.60 \mathrm{~nm}^{2}$ ). With $\epsilon=78.5$ this gives $\sigma \approx-0.27$ $\mathrm{C} \cdot \mathrm{m}^{-2}$ and $c_{\mathrm{s}}^{0} \approx 21 \mathrm{M}$. This value of the surface concentration is far above the saturation concentration of the channel (Fig. 1). Furthermore, at such a high concentration contact adsorption of ions at the membrane surface is likely to occur (see below).

The experimental findings are in qualitative agreement with the predictions of the Gouy-Chapman theory in so far as the single-channel conductance seems to approach a finite value at low ion concentration (Fig. 1). The reason for the slight decrease of $\Lambda$ at low concentration is not clear, however, and several possible explanations may be discussed. At low ion concentrations $c$ the surface potential becomes strongly negative (at $c=10 \mathrm{mM}$ a value of $\psi_{\mathrm{s}}=-197 \mathrm{mV}$ is 
calculated with $\sigma=-0.27 \mathrm{C} \cdot \mathrm{m}^{-2}$ ). According to Eqn. 4 this means that not only the $\mathrm{Cs}^{+}$concentration but also the concentration of $\mathrm{H}^{+}$is increased at the surface. This reduction in surface $\mathrm{pH}$ may lead to a partial protonation of the carboxyl groups of phosphatidylserine which in turn would decrease the density of surface charge. We consider this explanation as unlikely, since the behaviour of $\Lambda$ at low ion concentration was found to be rather insensitive to $\mathrm{pH}$ changes in the solution in the $\mathrm{pH}$ range between 6 and 9 [21].

Another possibility for the decrease of $\Lambda$ consists in a diffusion limitation of ion transport at low electrolyte concentrations. If the rate of ion transport through the channel is high, the diffusional flow of ions from the aqueous solution to the channel mouth may become rate limiting [29]. This effect should be more pronounced outside the saturation region of the channel that is, at low ion concentrations. The quantitative theory predicts that for a neutral diffusion limitation should become severe if the ratio $\Lambda / c$ is of the order of $1-10 \mathrm{~ns} / \mathrm{M}$ or larger [29]. For a cation-permeable channel in a negatively charged membrane, however, the effect of the finite diffusion rate is smaller since the cation concentration is increased in the space-charge layer at the membrane surface. Experimental values of $\Lambda / c$ for the phosphatidylserine membrane (Fig. 1) at low ion concentrations $\left(1^{--10} \mathrm{mM}\right)$ range between 4.2 and $9.6 \mathrm{nS} / \mathrm{M}$. For this reason it cannot be completely excluded that diffusion limitation has influence at, least in part, on the decrease of $\Lambda$ at low electrolyte concentrations.

A third contribution to the behaviour of $\Lambda(c)$ may result from an effect of lipid structure on the single-channel conductance. The structure of a charged bilayer if affected by the ionic composition of the aqueous phase. This fact is well documented by the observed dependence of transition temperature of charged lipids on the ionic environment [30]. It is therefore conceivable that the increase in lateral pressure of the phosphatidylserine bilayer at low ionic strength affects the structure and the dynamic properties of the channel. A question of a more general nature concerns the applicability of the GouyChapman treatment to the case of a channel embedded in a charged bilayer. Although several restrictive assumptions are introduced into the derivation of the Gouy-Chapman equations, the theory accounts surprisingly well for the experimental results, obtained with hydrophobic ions and alkali-ion carriers in lipid bilayers bearing a net charge [1-8]. If a channel is built into the bilayer, the structure of electric double-layer is perturbed, as the channel molecule replaces several lipid molecules. A more realistic treatment therefore should be based on a model where the mouth of the channel is located at the center of a circular disk free of fixed charges. If $r_{\mathrm{c}}$ is the radius of the uncharged disk embedded into an infinite plane of charge density $\sigma$, the electric potential $\psi_{\mathrm{c}}$ in the center of the disk is given by (Appendix A):

$\psi_{\mathrm{c}}=\psi_{\mathrm{s}} \cdot \exp \left(-r_{\mathrm{c}} / L_{\mathrm{D}}\right)$

where $\psi_{\mathrm{s}}$ is the surface potential of the unperturbed layer and $L_{\mathrm{D}}$ is the Debye length:

$L_{\mathrm{D}}=\frac{1}{F} \sqrt{\frac{\epsilon_{0} \epsilon R T}{2 c}}$ 
Eqn. 6 is strictly valid only for small potentials $\left(\left|\psi_{s}\right|<<R T / F\right)$; in this limit $\psi_{\text {s }}$ is equal to $\sigma L_{\mathrm{D}} / \epsilon_{0} \epsilon$. (At larger potentials numerical solutions for $\psi_{\mathrm{c}}$ have to be used.) Eqn. 6 shows that the electrical potential $\psi_{c}$ at the channel mouth may be considerably smaller than $\psi_{\mathrm{s}}$ unless $r_{\mathrm{c}}$ is of the same order or smaller than the Debye length $L_{\mathrm{D}}$. From the structure of the gramicidin channel [31] a value of $r_{\mathrm{c}} \approx 1.0 \mathrm{~nm}$ (corresponding to the outer radius of the channel) may be estimated. According to Eqn. 7 this means that $\psi_{\mathrm{c}} \approx \psi_{\mathrm{s}}$ for $c<0.05 \mathrm{M}$. As Eqn. 6 predicts that $\psi_{\mathrm{c}}$ approaches $\psi_{\mathrm{s}}$ for $c \rightarrow 0$, the drop of the channel conductance at small values of $c$ cannot be explained by the existence of a chargefree area around the channel mouth.

Two other effects which are not accounted for in the Gouy-Chapman treatment and which are of possivle relevance here are the effect of the discrete nature of fixed charges and the binding of counterions. Discrete charge effects at the membrane-solution interface have been analysed by Brown [32] for the linear range of the Poisson-Boltzmann equation and recently, for arbitrary potentials, by Sauvé and Okhi (submitted for publication). A numerical evaluation of the model of Sauvé and Okhi has been carried out assuming a hexagonal lattice of charges of radius $r_{0}=10 \mathrm{~nm}$, the channel mouth being located at the central (charge-free) lattice point [21]. Outside the lattice a diffuse distribution of surface charges has been assumed. This model seems to describe the data equally well, although the experimental results in the saturation region of the channel are not sensitive enough for a discrimination between the Gouy-Chapman treatment and the discrete-charge model.

A further possible effect which has to be discussed here is count rion binding at the charged interface. As mentioned earlier, the concentration of cations at the surface of a pure phosphatidylserine membrane is estimated, according to Eqn. 4, to be about $20 \mathrm{M}$. At this high concentration (which is nearly the concentration in a crystalline solid) part of the cations are probably bound to the membrane surface. Ion adsorption to a charged surface may be described by the model of Stern [33] in which the electric double-layer is dividided into an inner layer containing the adsorbed ions and an outer layer with a diffuse ion distribution. According to Stern the layer of adsorbed ions may be treated as a molecular capacitor in which the electrical potential drops linearly from the value $\psi_{s}$ at the surface to the value $\psi^{\prime}$ in the plane of the adsorbed counterions:

$\sigma=\frac{\epsilon_{0} \epsilon^{\prime}}{l}\left(\psi_{\mathrm{s}}-\psi^{\prime}\right)$

( $\epsilon^{\prime}$ is the dielectric constant of the Stern layer and $l$ is the distance from the center of the adsorbed ions to the surface). Neglecting specific adsorption and the presence of co-ions in the Stern layer, the charge density $\sigma^{\prime}$ of the contactabsorbed ions is given by

$\sigma^{\prime}=e_{0} N \frac{c \bar{V}}{c \bar{V}+\exp \left(F \psi^{\prime} / R T\right)}$

where $N$ is the maximum number of counterions which may be present in the Stern layer and $\bar{V}$ is the molar volume of the counterions $(c \bar{V}$ is the volume fraction of the counterions in the solution). The total charge in the diffuse ion- 
layer is electrically neutralized by the net surface charge density $\sigma-\sigma^{\prime}$ which is given by the Gouy-Chapman relation

$\sigma-\sigma^{\prime}=\sigma_{0} \sinh \left(F \psi^{\prime} / 2 R T\right)$

For the application of the Stern model an assumption concerning the entry rate of ions into the channel has to be introduced. The rate of entry may be set proportional to the ion concentration in the Stern layer. Another possibility consists in assuming that the entry rate is proportional to the concentration of cations in the diffuse part of the double layer, averaged over a layer of a certain thickness $\delta$ adjacent to the adsorption plane. A numerical evaluation of the Stern model and other models should take into account the geometry of the membrane surface around the channel mouth. As the length of the gramidicin channel is less than the membrane thickness, the membrane surface is curved in the vicinity of the channel $[19,35]$. This effect should lead to a higher charge density near the channel mouth than in the case of planar surface. Indeed, in experiments with phosphatidylserine membranes made by the Montal-Mueller technique [36], which have a reduced thickness, the conductance of the gramicidin channel was found to be smaller by about $20 \%$ (in $5 \mathrm{mM} \mathrm{CsCl}$ ) than the conductance in phosphatidylserine/ $n$-decane membranes (Apell, H.-J., unpublished data).

\section{Appendix A}

\section{Derivation of Eqn. 6}

We consider an uncharged circular disk of radius $r_{\mathrm{c}}$ embedded into a plane of charge density $\sigma$ (Fig. 3). The disk and the surface are in contact with the aqueous solution of a 1:1 electrolyte of concentration $c$. In order to calculate the electric potential $\psi$ as a function of the coordinate $x$ normal to the center of the disk, we first consider a point charge of magnitude $q$ in a homogeneous medium. For small values of the potential, where the PoissonBoltzmann equation can be used in the linearized form, the potential at a

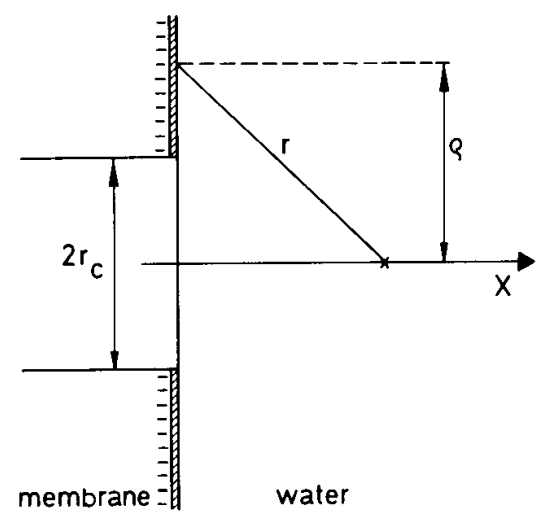

Fig. 3. Uncharged disk of radius $r_{c}$ embedded in an electrically charged plane. 
distance $r$ from the charge is given by [32]:

$\psi(r)=\frac{q}{4 \pi \epsilon_{0} \epsilon r} \exp \left(-r / L_{\mathrm{D}}\right)$

$\epsilon_{0}$ is the permittivity of free space, $\epsilon$ the dielectric constant of water and $L_{\mathbf{D}}$ the Debye length (Eqn. 7). If the charge $\mathrm{d} q=2 \pi \rho \sigma \mathrm{d} \rho$ is distributed over a ring of radius $\rho$ and width $\mathrm{d} \rho$ around the center of the disk (Fig. 3 ) the contribution of $\mathrm{d} q$ to the potential at point $x$ is

$\mathrm{d} \psi=\frac{\sigma \rho}{\epsilon_{0} \epsilon} \frac{\exp \left(-\sqrt{\rho^{2}+x^{2}} / L_{\mathrm{D}}\right)}{\sqrt{\rho^{2}+x^{2}}} \mathrm{~d} \rho$

Implicit in Eqn. A2 is the assumption that the electric field from the surface charges is virtually zero in the membrane phase. This assumption is reasonable as long as the dielectric constant of the membrane is small compared with $\epsilon$. Integration of Eqn. A2 between $\rho=r_{\mathrm{c}}$ and $\rho=\infty$ yields

$$
\begin{aligned}
\psi(x) & =\frac{\sigma L_{\mathrm{D}}}{\epsilon_{0} \epsilon}\left|-\exp \left(-\sqrt{\rho^{2}+x^{2}} / L_{\mathrm{D}}\right)\right|_{\rho=r_{\mathrm{c}}}^{\rho=\infty} \\
& =\frac{\sigma L_{\mathrm{D}}}{\epsilon_{0} \epsilon} \cdot \exp \left(--\sqrt{r_{\mathrm{c}}^{2}+x^{2}} / L_{\mathrm{D}}\right)
\end{aligned}
$$

For $x=0$, Eqn. A3 reduces to Eqn. 6 .

\section{Acknowledgements}

We thank Dr. R. Sauvé for interesting discussions and for providing a computer program for the numerical analysis of the discrete-charge model and Dr. E. Gross (Bethesda) for the gift of gramicidin A. This work has been financially supported by Deutsche Forschungsgemeinschaft (Sonderforschungsbereich 138).

\section{References}

1 McLaughlin, S. (1977) in Current Topics in Membranes and Transport (Bronner, F. and Kleinzeller, A., eds.), Vol. 9, Academic Press, New York

2 Lesslauer, W., Richter, J. and Läuger, P. (1967) Nature 213, 1224-1226

3 Hopfer, U., Lehninger, A.L. and Lennarz, W.J. (1970) J. Membrane Biol. 3, 142-155

4 McLaughlin, S. (1972) J. Membrane Biol. 9, 361-372

5 Foster, M. and McLaughlin, S. (1974) J. Membrane Biol. 17, 155-180

6 Benz, R. and Läuger, P. (1977) Biochim. Biophys. Acta 468, 245-258

7 McLaughlin, S.G.A., Szabo, G., Eisenman, G. and Ciani, S.M. (1970) Proc. Natl. Acad. Sci. U.S. 67 , $1268-1275$

8 McLaughlin, S.G.A., Szabo, G. and Eisenman, G. (1971) J. Gen. Physiol. 58, 667-687

9 Muller, R.U. and Finkelstein, A. (1972) J. Gen. Physiol. 60, 285-306

10 Finkelstein, A. and Holz, R. (1973) in Membranes, A Series of Advances (Eisenman, G., ed.), Vol. 2, pp. 377-408, Marcel Dekker. New York

11 Bamberg, E. and Janko, K. (1976) Biochim. Biophys. Acta 426, 447-450

12 Ermishkin, L.N., Kasumov, Kh.M. and Potselnyev, V.M. (1977) Biochim. Biophys. Acta 470, 357367

13 Bamberg, E., Alpes, H., Apell, H.-J., Benz, R., Janko, K., Kolb, H.-A. and Läuger, P. (1977) in Biochemistry of Membrane Transport, FEBS Symposium No. 42 (Semenza, G. and Carafoli, E., eds.), pp. 179-201, Springer, Berlin 
14 Hille, B. (1968) J. Gen. Physiol. 51, 221-236

15 Drouin, H. and Neumcke, B. (1974) Pflügers Arch. 351, 207--229

16 Hille, B., Woodhull, A.M. and Shapiro, B.I. (1975) Philos, Trans, Roy. Soc. B270, 301-318

17 Janko, K. and Benz, R. (1977) Biochim. Biophys. Acta 470, 8-16

18 Läuger, P., Lesslauer, W., Marti, E. and Richter, J. (1967) Biochim. Biophys. Acta 135, 20-32

19 Hladky, S.B, and Haydon, D.A. (1972) Biochim. Biophys. Acta 274, 294-312

20 Bamberg, E., Noda, K., Gross, E. and Läuger, P. (1976) Biochim. Biophys. Acta 419, 223-228

21 A pell, H.-J. (1978) Dissertation, Universität Konstanz

22 Neher, E., Sandblom, J. and Eisenman, G. (1978) J. Membrane Biol. 40, 97-116

23 Eisenman, G., Sandblom, J. and Neher, E. (1978) Biophys. J. 22, 307-340

24 Levitt, D.G. (1978) Biophys. J. 22, 209-219

25 Levitt, D.G. (1978) Biophys. J. 22, 221-248

26 A pell, H.-J., Bamberg, E., Alpes, H. and Läuger, P. (1977) J. Membrane Biol. 31, 171-188

27 Aveyard, R. and Haydon, D.A. (1973) An Introduction to the Principles of Surface Chemistry, Cambridge University Press

28 Hladky, S.B. and Haydon, D.A. (1973) Biochim. Biophys. Acta 318, 464-468

29 Läuger, P. (1976) Biochim. Biophys. Acta 455, 493-509

30 Träuble, H. and Eibl, H. (1974) Proc. Natl. Acad. Sci. U.S. 71, 214-219

31 Urry, D.W. (1971) Proc. Natl. Acad. Sci. U.S. 68, 672-676

32 Brown, R.H., Jr. (1974) Prog. Biophys. Mol. Biol, 28, 341-370

33 Stern, O. (1924) Z. Elecktrochem. 30, 508-516

34 Bamberg, E. and Läuger, P. (1977) J. Membrane Biol. 35, 351-375

35 Haydon, D.A., Hendry, B.M., Levinson, S.R. and Requena, J. (1977) Nature 268, 356-358

36 Montal, M. and Mueller, P. (1972) Proc. Natl. Acad. Sci. U.S. 69, 3561-3566 